\title{
PRESCRIBING CURVATURE WITH NEGATIVE TOTAL CURVATURE ON OPEN RIEMANN SURFACES
}

\author{
JUNJIE TANG
}

(Communicated by Peter Li)

\begin{abstract}
On open Riemann surfaces, we obtain a necessary and sufficient condition for conformal metrics to have prescribed curvature and the desired singularities leading to negative total curvature.
\end{abstract}

\section{INTRODUCTION}

Suppose $M$ is a compact Riemann surface with Euler characteristic $\chi(M)$. Let $g$ be a compatible metric with its Gaussian curvature $K$. The problem of prescribed curvature is as follows: for a given function $\widetilde{K} \in C^{\infty}(M)$, is there a metric $\tilde{g}$ that is conformal to $g$ and has $\widetilde{K}$ as its curvature (cf. [KW])? If we write $\tilde{g}=e^{2 u} g$, this problem is equivalent to solving the elliptic equation

$$
\Delta u+\widetilde{K} e^{2 u}=K
$$

on $M$, where $\Delta$ is the Laplacian under the metric $g$. If we integrate (1) over $M$ we find that the total curvature of $\tilde{g}$ agrees with that of $g$ and is fixed by the Gauss-Bonnet Theorem to be $2 \pi \chi(M)$.

Let $\Gamma$ be a finite set $\left\{p_{1}, \ldots, p_{n}\right\}$ of points on $M$, and let $\widehat{M}=M \backslash \Gamma$. Then $\widehat{M}$ is an open surface. The problem of prescribed curvature on $\widehat{M}$ can be stated as follows: for a given function $\widetilde{K} \in C(\widehat{M})$, does there exist a metric $\tilde{g}$ on $\widehat{M}$ that is conformal to $g$ and has $\widetilde{K}$ as its curvature on $\widehat{M}$ ? In this case, we should find a solution of the equation (1) on $\widehat{M}$. Since $\widehat{M}$ is noncompact the total curvature of $\tilde{g}$ need not agree with that of $g$; the difference depends on the asymptotic behaviour of the solution $u$ near the points $p_{i}$. In this paper, the asymptotic behaviour of the solution $u$ contributes singularities to $\tilde{g}$.

As a special case we may take $\widehat{M}=\mathbf{R}^{2}=S^{2} \backslash\{p\}$. Several authors have considered the prescribed curvature problem on $\mathbf{R}^{2}$ (cf. [N, M2, M3, A, CN]). A good survey is due to Kazdan (cf. [K]). Recently, many of the results on $\mathbf{R}^{2}$ have been extended to the more general open surfaces $\widehat{M}$ by Hulin and Troyanov [HT] and McOwen [M1].

Received by the editors April 17, 1991.

1980 Mathematics Subject Classification (1985 Revision). Primary 35J60; Secondary 53A30.

Key words and phrases. Riemann surface, conformal metric, prescribed curvature, asymptotic behaviour. 
For example, $\mathrm{Ni}[\mathrm{N}]$ and McOwen [M3] considered the problem of prescribed curvature with negative total curvature on $\mathbf{R}^{2}$. They found that if $\widetilde{K} \leq 0$ and $\widetilde{K}=O\left(|x|^{-l}\right)$ for $l>2$, then there are conformal metrics with $\overline{\widetilde{K}}$ as its curvature and negative total curvature on $\mathbf{R}^{2}$. This result was extended to the more general surfaces $\widehat{M}$ in [HT, M1].

As another example, in [M2] McOwen used the variational method to obtain results on positive total curvature on $\mathbf{R}^{2}$; but, in addition, he observed that a necessary and sufficient condition for a conformal metric on $\mathbf{R}^{2}$ to have curvature $\widetilde{K}=O\left(|x|^{-l}\right)$ for $l>2$ and zero total curvature is:

(i) $\widetilde{K}$ changes sign on $\mathbf{R}^{2}$,

(ii) $\int \widetilde{K} d x<0$.

These results were extended to the more general surfaces $\widehat{M}$ in [HT]; in particular, the necessary and sufficient conditions for $\widetilde{K}$ to be the curvature of a conformal metric with zero total curvature become

(i*) $\widetilde{K}$ changes sign on $\widehat{M}$,

(ii*) $\int \widetilde{K} d A_{g_{\alpha}}<0$,

where $g_{\bar{\alpha}}$ is a flat conformal metric representing the divisor $\bar{\alpha}$ on $M$ (cf. $\S 1$, Definition and Proposition).

In this paper, we shall study the prescribed curvature problem with negative total curvature, but removing the assumption $\widetilde{K} \leq 0$ that exists in all previous works (cf. [N, M1, M3, HT]). We shall find that the condition (ii ${ }^{*}$ ) is as decisive for negative total curvature as it was for zero total curvature: if (ii ${ }^{*}$ ) holds under some flat conformal metric $g_{\bar{\alpha}}$ representing the divisor $\bar{\alpha}$ on $M$, then there are conformal metrics with $\widetilde{K}$ as their curvature and negative total curvature; in other words, condition $\left(\right.$ ii $\left.^{*}\right)$ implies that the equation (1) on $\widehat{M}$ has solutions with the prescribed asymptotic behaviour near the points $p_{i}$. Moreover, condition $\left(\mathrm{ii}^{*}\right)$ is also necessary for the existence of solutions of (1) with the desired asymptotic behaviour near the points $p_{i}$ (cf. Theorems A, B). In the special case $\mathbf{R}^{2}=S^{2} \backslash\{p\}$, we obtain condition (ii*) as a necessary and sufficient condition on $\mathbf{R}^{2}$ (cf. Corollary). This result was not obtained in the earlier work on $\mathbf{R}^{2}$.

\section{MAIN THEOREMS}

Throughout this paper, without loss of generality, we assume that the metric $g$ is Euclidean in a neighborhood of each point $p_{i}$ (cf. [M1]). Denote $r_{i}=$ $\operatorname{dist}\left(x, p_{i}\right)$ under the metric $g$. Following Hulin and Troyanov (cf. [HT]), we shall use the following terminology.

Definition. On a compact Riemann surface $M$, a metric $g_{\alpha}$ is said to represent the divisor $\alpha:=\sum_{i=1}^{n} \alpha_{i} p_{i}, \alpha_{i} \in \mathbf{R}$, if there is $\nu \in C^{\infty}(\widehat{M}) \cap H_{2}^{p}(M)$ for some $p>1$ such that $g_{\alpha}=e^{2 \nu} r_{i}^{2 \alpha_{i}} g$ in a neighborhood of each point $p_{i}$. In this case, the Euler characteristic $\chi(M, \alpha)$ of $(M, \alpha)$ is defined by

$$
\chi(M, \alpha)=\chi(M)+\sum_{i=1}^{n} \alpha_{i} .
$$

Remark. By $\nu \in C^{\infty}(\widehat{M}) \cap H_{2}^{p}(M)$ we mean that $\nu \in H_{2}^{p}(M)$ and is $C^{\infty}$ on 
$\widehat{M}$. Also, the more general conditions $\nu \in L^{1}(M)$ and $\Delta \nu \in L^{1}(M)$ (weakly) that are used in [HT] will not be necessary in our case.

We first observe that if $\chi(M, \bar{\alpha})=0$ then we can represent $\bar{\alpha}$ by a flat conformal metric. This was observed by Hulin and Troyanov (cf. [HT, Theorem 7.1]), but we shall give a proof in $\S 2$ for the sake of completeness.

Proposition. Let $M$ be a compact Riemann surface, let $\Gamma=\left\{p_{1}, \ldots, p_{n}\right\} \subset M$, and let $\widehat{M}=M \backslash \Gamma$. If $\bar{\alpha}:=\sum_{i=1}^{n} \bar{\alpha}_{i} p_{i}$ is a divisor with $\chi(M, \bar{\alpha})=0$, then there exists a flat conformal metric $g_{\bar{\alpha}}=e^{2 u_{1}} g$ representing $\bar{\alpha}$. Moreover, $g_{\bar{\alpha}}$ is unique up to homothety (multiplication of $g_{\bar{\alpha}}$ by a positive constant).

Next, we investigate the role $g_{\bar{\alpha}}$ plays in representing a divisor $\alpha$ by a conformal metric $g_{\alpha}=e^{2 u} g$. The following result provides a necessary condition. For the sake of convenience, we define for any two divisors $\alpha:=\sum_{i=1}^{n} \alpha_{i} p_{i}$ and $\beta:=\sum_{i=1}^{n} \beta_{i} p_{i}$ having same support $\Gamma$,

(a) $|\alpha-\beta|=\max _{1 \leq i \leq n}\left|\alpha_{i}-\beta_{i}\right|$;

(b) $\alpha \leq \beta$, if $\alpha_{i} \leq \beta_{i}$ for each $i$.

Theorem A. Suppose $M$ is a compact Riemann surface. Let $\Gamma=\left\{p_{1}, \ldots, p_{n}\right\} \subset$ $M$ and $\widehat{M}=M \backslash \Gamma$. Suppose $\widetilde{K} \in C^{\infty}(\widehat{M})$ satisfies $\widetilde{K}=O\left(r_{i}^{l_{i}}\right)$ as $r_{i} \rightarrow 0$ and $\sum_{i=1}^{n} l_{i}>2(\chi(M)-n)=2 \chi(\widehat{M})$. If there exists a conformal metric $g_{\alpha}$ representing a divisor $\alpha:=\sum_{i=1}^{n} \alpha_{i} p_{i}$ with $\widetilde{K}$ as its curvature, if $\chi(M, \alpha)<0$, and if $\alpha_{i}>\left(l_{i}+2\right) / 2$ for each $i$, then for any divisor $\bar{\alpha}:=\sum_{i=1}^{n} \bar{\alpha}_{i} p_{i}$ with $\chi(M, \bar{\alpha})=0$ and $\alpha \leq \bar{\alpha}$, we have $\int_{M} \widetilde{K} d A_{g_{\bar{\alpha}}}<0$, where $g_{\bar{\alpha}}$ is a flat conformal metric representing $\bar{\alpha}$ and $d A_{g_{\bar{\alpha}}}$ is the area element under the metric $g_{\bar{\alpha}}$.

Finally, we show that the condition $\int_{M} \widetilde{K} d A_{g_{\tilde{\alpha}}}<0$ is sufficient to represent divisors $\alpha$ near $\bar{\alpha}$ by conformal metrics $g_{\alpha}$ having curvature $\widetilde{K}$.

Theorem B. Suppose $M$ is a compact Riemann surface. Let $\Gamma=\left\{p_{1}, \ldots, p_{n}\right\} \subset$ $M$ and $\widehat{M}=M \backslash \Gamma$. Suppose $\widetilde{K} \in C^{\infty}(\widehat{M})$ satisfies $\widetilde{K}=O\left(r_{i}^{l_{i}}\right)$ as $r_{i} \rightarrow 0$ and $\sum_{i=1}^{n} l_{i}>2(\chi(M)-n)=2 \chi(\widehat{M})$. If $\int_{M} \widetilde{K} d A_{g_{\tilde{\alpha}}}<0$ for a flat conformal metric $g_{\bar{\alpha}}$ representing a divisor $\bar{\alpha}:=\sum_{i=1}^{n} \bar{\alpha}_{i} p_{i}$ with $\chi(M, \bar{\alpha})=0$, and if $\bar{\alpha}_{i}>-\left(l_{i}+2\right) / 2$ for each $i$, then there is $\varepsilon>0$ such that for any divisor $\alpha:=\sum_{i=1}^{n} \alpha_{i} p_{i}$ satisfying $\chi(M, \alpha)<0$ and for $|\alpha-\bar{\alpha}|<\varepsilon$, there exists $a$ conformal metric $g_{\alpha}$ representing the divisor $\alpha$ with $\widetilde{K}$ as its curvature.

Remark. In Theorems A and B, there is a restriction on the $l_{i}$ 's implied by $\chi(M, \alpha)<0$ and $\alpha_{i}>-\left(l_{i}+2\right) / 2$ for each $i$; namely, $\sum_{i=1}^{n} l_{i}>2(\chi(M)-n)$. The total curvature $\tilde{k}$ under the conformal metric $g_{\alpha}$ is

$$
\tilde{k}=\int_{M} \widetilde{K} e^{2 u} d A_{0}=\int_{M}(K-\Delta u) d A_{0}=2 \pi \chi(M, \alpha)
$$

where $d A_{0}$ is the area element under the metric $g$. Thus, $\tilde{k}<0$. By Proposition, condition $\left(\mathrm{ii}^{*}\right)$ in Theorems $\mathrm{A}$ and $\mathrm{B}$ does not depend on which flat conformal metric we choose in the homothety class.

If we apply Theorems A and B to the case $\widehat{M}=S^{2} \backslash\{p\}$, then we obtain the following necessary and sufficient condition for solving the prescribed curvature problem on $\mathbf{R}^{2}$. 
Corollary. Suppose $\widetilde{K} \in C^{2}\left(R^{2}\right)$ satisfies the condition that $\widetilde{K}=O\left(|x|^{-l}\right)$ as $|x| \rightarrow \infty$ for some $l>2$. Then there exists $\varepsilon>0$ such that for any $\lambda$, $0<\lambda<\varepsilon$, the equation

$$
\Delta u+\widetilde{K} e^{2 u}=0 \text { in } \mathbf{R}^{2}
$$

has a solution $u$ with the asymptotic behaviour

$$
u=\lambda \log |x|+c+o(1) \quad \text { as }|x| \rightarrow \infty
$$

where $c$ is a constant if and only if $\int_{R^{2}} \widetilde{K} d x<0$.

\section{Proofs of THE THEOREMS}

In this section, we first give a proof of Proposition that is slightly different from the one in $[\mathrm{HT}]$.

Proof of Proposition. Choose $V_{\bar{\alpha}} \in C^{\infty}(\widehat{M})$ with $V_{\bar{\alpha}}=\bar{\alpha}_{i} \log r_{i}+\nu$ in a neighborhood of each point $p_{i}$, where $\nu \in C^{\infty}(\widehat{M}) \cap H_{2}^{p}(M)$ for some $p>1$. Note that $K-\Delta V_{\bar{\alpha}}$ can be extended in $L^{p}(M) \cap C^{\infty}(\widehat{M})$ and

$$
\int_{M}\left(K-\Delta V_{\bar{\alpha}}\right) d A_{0}=2 \pi\left(\chi(M)+\sum_{i=1}^{n} \bar{\alpha}_{i}\right)=0
$$

where $d A_{0}$ is the area element under the metric $g$. Thus there exists $u_{0} \in$ $H_{2}^{p}(M) \cap C^{\infty}(\widehat{M})$ such that $\Delta u_{0}=K-\Delta V_{\bar{\alpha}}$. If $\nu \in C^{\infty}(M)$ then $u_{0} \in C^{\infty}(M)$. Let $u_{1}=u_{0}+V_{\bar{\alpha}}$. So $g_{\bar{\alpha}}=e^{2 u_{1}} g$ is a flat conformal metric representing $\bar{\alpha}$. For any flat conformal metric $e^{2 w_{1}} g$ representing $\bar{\alpha}$, we have $u_{1}-w_{1} \in$ $H_{2}^{p}(M) \cap C^{\infty}(\widehat{M}) \subset C(M) \cap C^{\infty}(\widehat{M})$. Since $\Delta\left(u_{1}-w_{1}\right)=0$, so $u_{1}-w_{1}$ is a constant.

Then we prove our necessary condition in order for the function $\widetilde{K}$ to be the curvature of a conformal metric representing the divisor $\alpha$.

Proof of Theorem A. Let $g_{\alpha}=e^{2 u} g$ be a conformal metric representing $\alpha$ with $\widetilde{K}$ as its curvature, and let $g_{\bar{\alpha}}=e^{2 u_{1}} g$ be a flat conformal metric representing $\bar{\alpha}$ with $\chi(M, \bar{\alpha})=0$ and $\alpha \leq \bar{\alpha}$. Thus

$$
\begin{gathered}
\Delta u+\widetilde{K} e^{2 u}=K, \\
\Delta u_{1}=K, \\
\widetilde{K} e^{2 u_{1}}=-\Delta\left(u-u_{1}\right) e^{-2\left(u-u_{1}\right)} \quad \text { on } \widehat{M} .
\end{gathered}
$$

By the assumption, $\int_{M} \widetilde{K} e^{2 u_{1}} d A_{0}$ is finite and

$$
\int_{M} \widetilde{K} e^{2 u_{1}} d A_{0}=-\int_{M} \Delta\left(u-u_{1}\right) e^{-2\left(u-u_{1}\right)} d A_{0} .
$$

Let $B_{\varepsilon}\left(p_{i}\right)=\left\{x \in M: \operatorname{dist}\left(x, p_{i}\right)<\varepsilon\right\}$ and $B_{\varepsilon}(\Gamma)=\bigcup_{i=1}^{n} B_{\varepsilon}\left(p_{i}\right)$. By the 
Green's formula,

$$
\begin{aligned}
\int_{M} \Delta( & \left.u-u_{1}\right) e^{-2\left(u-u_{1}\right)} d A_{0} \\
=\lim _{\varepsilon \rightarrow 0} & \int_{M \backslash B_{\varepsilon}(\Gamma)} \Delta\left(u-u_{1}\right) e^{-2\left(u-u_{1}\right)} d A_{0} \\
=\lim _{\varepsilon \rightarrow 0} & {\left[\sum_{i=1}^{n} \int_{\partial B_{\varepsilon}\left(p_{i}\right)} \frac{\partial\left(u-u_{1}\right)}{\partial n_{i}} e^{-2\left(u-u_{1}\right)} d s_{i}\right.} \\
& \left.\quad-\int_{M \backslash B_{\varepsilon}(\Gamma)} \nabla\left(u-u_{1}\right) \nabla e^{-2\left(u-u_{1}\right)} d A_{0}\right]
\end{aligned}
$$

where $n_{i}$ is the unit normal towards $p_{i}$ on $\partial B_{\varepsilon}\left(p_{i}\right)$ and $d s_{i}$ is the length element under the metric on $\partial B_{\varepsilon}\left(p_{i}\right)$ induced by the metric $g$. If $\alpha_{i}<\bar{\alpha}_{i}$, as $\varepsilon \rightarrow 0$

$$
\left|\int_{\partial B_{\varepsilon}\left(p_{i}\right)} \frac{\partial\left(u-u_{1}\right)}{\partial n_{i}} e^{-2\left(u-u_{1}\right)} d s_{i}\right| \leq M_{i} \varepsilon^{2\left(\bar{\alpha}_{i}-\alpha_{i}\right)} \rightarrow 0
$$

for some $M_{i}>0$. If $\alpha_{i}=\bar{\alpha}_{i}$, as $\varepsilon \rightarrow 0$

$$
\int_{\partial B_{\varepsilon}\left(p_{i}\right)} \frac{\partial\left(u-u_{1}\right)}{\partial n_{i}} e^{-2\left(u-u_{1}\right)} d s_{i}=o(1) \rightarrow 0 .
$$

Hence

$$
\int_{M} \Delta\left(u-u_{1}\right) e^{-2\left(u-u_{1}\right)} d A_{0}=2 \int_{M}\left|\nabla\left(u-u_{1}\right)\right|^{2} e^{-2\left(u-u_{1}\right)} d A_{0}>0,
$$

which implies $\int_{M} \tilde{K} e^{2 u_{1}} d A_{0}<0$. Therefore, $\int_{M} \tilde{K} d A_{g_{\bar{\alpha}}}<0$.

In order to prove the existence of the solution $u$ of equation (1) with the desired asymptotic behaviour near the points $p_{i}$, we first rewrite equation (1), then construct upper and lower solutions. Suppose $V_{i} \in C^{\infty}\left(M \backslash\left\{p_{i}\right\}\right)$ satisfies $V_{i}=\log r_{i}$ in a neighborhood of $p_{i}$. Let $V_{\alpha}=\sum_{i=1}^{n} \alpha_{i} V_{i}$ for a divisor $\alpha:=$ $\sum_{i=1}^{n} \alpha_{i} p_{i}$. Then $V_{\alpha} \in C^{\infty}(\widehat{M})$ satisfies $V_{\alpha}=\alpha_{i} \log r_{i}+V_{0}$ in a neighborhood of each point $p_{i}$, where $V_{0} \in C^{\infty}(M)$. Let $V_{\bar{\alpha}}=\sum_{i=1}^{n} \bar{\alpha}_{i} V_{i}$ for a divisor $\bar{\alpha}:=\sum_{i=1}^{n} \bar{\alpha}_{i} p_{i}$ with $\chi(M, \bar{\alpha})=0$. By the proof of Proposition, there is a unique $u_{0} \in C^{\infty}(M)$ up to an additive constant such that $\Delta u_{0}=K-\Delta V_{\bar{\alpha}}$ on $\widehat{M}$. Since

$K-\Delta V_{\alpha}-\frac{2 \pi \chi(M, \alpha)}{\operatorname{area}(M)} \in C^{\infty}(M)$ and $\int_{M}\left[K-\Delta V_{\alpha}-\frac{2 \pi \chi(M, \alpha)}{\operatorname{area}(M)}\right] d A_{0}=0$,

there is a unique $U_{\alpha} \in C^{\infty}(M)$ up to an additive constant such that

$$
\Delta U_{\alpha}=K-\Delta V_{\alpha}-2 \pi \chi(M, \alpha) / \operatorname{area}(M) \text {. }
$$

We may choose $\int_{M} u_{0} d A_{0}=0$, and $\int_{M} U_{\alpha} d A_{0}=0$. Let $u=W+U_{\alpha}+V_{\alpha}$ on $\widehat{M}$. Then equation (1) can be transformed into

$$
\Delta W+\widetilde{K}_{\alpha} e^{2 W}=c_{\alpha}
$$

on $\widehat{M}$, where $\widetilde{K}_{\alpha}=\widetilde{K} e^{2\left(U_{n}+V_{\alpha}\right)}$ and $c_{\alpha}=2 \pi \chi(M, \alpha) / \operatorname{area}(M)$. In order to solve equation (1) for $u$ with $u=\alpha_{i} \log r_{i}+\nu$ in a neighborhood of each point 
$p_{i}$, where $\nu \in C^{\infty}(\widehat{M}) \cap H_{2}^{p}(M)$ for some $p>1$, we ought to solve equation (2) for $W \in C^{\infty}(\widehat{M}) \cap H_{2}^{p}(M)$. In [KW, $\left.\S 10\right]$, Kazdan and Warner discussed the equation $\Delta u=c-h e^{u}$ with $c<0$ in the $C^{\infty}(M)$ category. Here in equation (2), $\widetilde{K}_{\alpha} \in L^{p}(M)$ for some $p>1$. So we shall modify the approach in [KW] and find a solution of equation (2) in $C^{\infty}(\widehat{M}) \cap H_{2}^{p}(M)$. To prove Theorem B, we introduce the following lemmas.

Lemma 1. Suppose $\widetilde{K} \in C^{\infty}(\widehat{M})$ is not identically zero, satisfies $\widetilde{K}=O\left(r_{i}^{l_{i}}\right)$ as $r_{i} \rightarrow 0$, and $\sum_{i=1}^{n} l_{i}>2(\chi(M)-n)$. Let $W^{+} \in C^{\infty}(\widehat{M})$ be a bounded upper solution of equation (2), i.e.,

$$
\Delta W^{+}+\widetilde{K}_{\alpha} e^{2 W^{+}} \leq c_{\alpha} \text { on } \widehat{M} .
$$

If the divisor $\alpha:=\sum_{i=1}^{n} \alpha_{i} p_{i}$ satisfies $\chi(M, \alpha)<0$ and $\alpha_{i}>-\left(l_{i}+2\right) / 2$ for each $i$, then there exists a bounded lower solution $W^{-} \in C^{\infty}(\widehat{M})$, i.e.,

$$
\Delta W^{-}+\widetilde{K}_{\alpha} e^{2 W^{-}} \geq c_{\alpha}
$$

and $W^{-} \leq W^{+}$on $\widehat{M}$. In this case, equation (2) has a bounded solution $W \in C^{\infty}(\widehat{\widehat{M}}) \cap H_{2}^{p}(M)$ for some $p>1$.

Proof. Let $h_{\alpha}=\left|\widetilde{K}_{\alpha}\right|$. Then $\int_{M} h_{\alpha} d A_{0}$ is finite and positive. Let

$$
\bar{f}_{\alpha}=c_{\alpha}-2 \pi \chi(M, \alpha) h_{\alpha} / \int_{M} h_{\alpha} d A_{0}
$$

Since $\alpha_{i}>-\left(l_{i}+2\right) / 2$ for each $i$, so $\bar{f}_{\alpha} \in L^{p}(M)$ for some $p>1$. Note that $\int_{M} \bar{f}_{\alpha} d A_{0}=0$. Thus there exists $W_{1} \in H_{2}^{p}(M) \subset C^{\beta}(M)$ for $0<\beta<2-2 / p$ such that $\Delta W_{1}=\bar{f}_{\alpha}$ on $M$. Hence $W_{1} \in C^{\infty}(\widehat{M}) \cap C(M)$. Let $W^{-}=W_{1}-\lambda$ for some positive constant $\lambda$. We have

$$
\begin{aligned}
\Delta W^{-}+\widetilde{K}_{\alpha} e^{2 W^{-}} & =c_{\alpha}-\frac{2 \pi \chi(M, \alpha) h_{\alpha}}{\int_{M} h_{\alpha} d A_{0}}+\widetilde{K}_{\alpha} e^{2 W_{1}-2 \lambda} \\
& \geq c_{\alpha}-h_{\alpha}\left[\frac{2 \pi \chi(M, \alpha)}{\int_{M} h_{\alpha} d A_{0}}+e^{2 W_{1}-2 \lambda}\right] \geq c_{\alpha}
\end{aligned}
$$

and $W^{-} \leq W^{+}$if a sufficiently large $\lambda$ is chosen. By the process in [M4] and [M1], it is easy to see the existence of a solution $W \in C^{\infty}(\widehat{M}) \cap H_{2}^{p}(M)$ to equation (2).

Lemma 2. Let $U \in H_{2}^{p}(M)$ with $\int_{M} U d A_{0}=0$ for $p>1$. Then

$$
|U|_{C^{\beta}} \leq d\|\Delta U\|_{p} \text {, }
$$

where $0<\beta<2-2 / p$ and $d$ is a constant independent of $U$.

Proof. This follows from the Sobolev's imbedding theorem and the standard elliptic estimates.

Lemma 3. Suppose $\widetilde{K} \in C^{\infty}(\widehat{M})$ satisfies $\widetilde{K}=O\left(r_{i}^{l_{i}}\right)$ as $r_{i} \rightarrow 0$ and $\sum_{i=1}^{n} l_{i}>$ $2(\chi(M)-n)$. Let the divisor $\bar{\alpha}:=\sum_{i=1}^{n} \bar{\alpha}_{i} p_{i}$ satisfy $\chi(M, \bar{\alpha})=0$ and $\bar{\alpha}_{i}>$ $-\left(l_{i}+2\right) / 2$ for each $i$. For any divisor $\alpha:=\sum_{i=1}^{n} \alpha_{i} p_{i}$ satisfying $\alpha_{i}>-\left(l_{i}+2\right) / 2$ for each $i$, let $h_{\alpha}=\left|\widetilde{K}_{\alpha}\right|$ and $C_{\alpha}=\int_{M} \widetilde{K}_{\alpha} d A_{0}$. Then we have

$$
C_{\alpha} \rightarrow \int_{M} \tilde{K} d A_{g_{\alpha}}
$$


and

$$
\int_{M} h_{\alpha} d A_{0} \rightarrow \int_{M}|\widetilde{K}| d A_{g_{\bar{\alpha}}} \quad \text { as } \alpha \rightarrow \bar{\alpha}
$$

where $g_{\bar{\alpha}}$ is a flat conformal metric representing $\bar{\alpha}$.

Proof. Claim that $U_{\alpha} \rightarrow u_{0}$ as $\alpha \rightarrow \bar{\alpha}$. Notice that

$$
\Delta\left(U_{\alpha}-u_{0}\right)=f_{\alpha} \text { with } \int_{M}\left(U_{\alpha}-u_{0}\right) d A_{0}=0,
$$

where $f_{\alpha}=-\Delta\left(V_{\alpha}-V_{\bar{\alpha}}\right)-c_{\alpha}$, and $f_{\alpha} \rightarrow 0$ as $\alpha \rightarrow \bar{\alpha}$. By Lemma 2,

$$
\left|U_{\alpha}-u_{0}\right|_{C^{\beta}} \leq d\left\|f_{\alpha}\right\|_{2} \text { for } 0<\beta<1 \text {. }
$$

Hence, $U_{\alpha} \rightarrow u_{0}$ as $\alpha \rightarrow \bar{\alpha}$. Thus, $\widetilde{K}_{\alpha}=\widetilde{K} e^{2\left(U_{\alpha}+V_{\alpha}\right)}$ converges to $\widetilde{K} e^{2\left(u_{0}+V_{\tilde{\alpha}}\right)}$ pointwise on $\widehat{M}$ as $\alpha \rightarrow \bar{\alpha}$. By Dominated Convergence Theorem, we have

$$
C_{\alpha} \rightarrow \int_{M} \widetilde{K} d A_{g_{\bar{\alpha}}}
$$

and

$$
\int_{M} h_{\alpha} d A_{0} \rightarrow \int_{M}|\widetilde{K}| d A_{g_{\bar{\alpha}}} \text { as } \alpha \rightarrow \bar{\alpha},
$$

where $g_{\bar{\alpha}}=e^{2\left(u_{0}+V_{\tilde{\alpha}}\right)} g$. From the proof of Proposition, we know that $g_{\bar{\alpha}}$ is a flat conformal metric representing $\bar{\alpha}$.

Recall that we want to solve equation (2). Using the condition $\int \widetilde{K} d A_{g_{\bar{\alpha}}}<0$ and Lemma 3, we shall construct a bounded upper solution $W^{+}$of equation (2). From Lemma 1, it follows that equation (2) admits a bounded solution, thereby proving Theorem $\mathbf{B}$.

Proof of Theorem B. Let $a_{\alpha}=\sqrt{|\alpha-\bar{\alpha}|}$, and observe

$$
|\chi(M, \alpha)| \leq \sum_{i=1}^{n}\left|\alpha_{i}-\bar{\alpha}_{i}\right| \leq n a_{\alpha}^{2}, \quad\left|c_{\alpha}\right| \leq \frac{2 \pi n a_{\alpha}^{2}}{\operatorname{area}(M)} .
$$

Now set

$$
\hat{f}_{\alpha}=\left[C_{\alpha}-\frac{2 \pi \chi(M, \alpha)}{a_{\alpha}}\right] \frac{h_{\alpha}}{\int_{M} h_{\alpha} d A_{0}}+\frac{c_{\alpha}}{a_{\alpha}}-\widetilde{K}_{\alpha} .
$$

Note that $\hat{f}_{\alpha} \in L^{p}(M)$ for some $p>1$ and $\int_{M} \hat{f}_{\alpha} d A_{0}=0$. Then there exists $W_{\alpha} \in H_{2}^{p}(M)$ such that $\Delta W_{\alpha}=\hat{f}_{\alpha}$ with $\int_{M} W_{\alpha} d A_{0}=0$. But $H_{2}^{p}(M) \subset C^{\beta}(M)$ for $0<\beta<2-2 / p$, together with local regularity imply $W_{\alpha} \in C^{\infty}(\widehat{M}) \cap C(M)$. From Lemma 2, $\left|W_{\alpha}\right|_{C^{\beta}} \leq d\left\|\hat{f}_{\alpha}\right\|_{p}$ for $0<\beta<2-2 / p$. By Lemma 3 , $\left\|\hat{f}_{\alpha}\right\|_{p}$ is bounded as $\alpha \rightarrow \bar{\alpha}$. Thus $W_{\alpha}$ is bounded as $\alpha \rightarrow \bar{\alpha}$. Let $W^{+}=a_{\alpha} W_{\alpha}+b_{\alpha}$, and $e^{2 b_{\alpha}}=a_{\alpha}$. From Lemma 3, we have

$$
C_{\alpha} \rightarrow \int_{M} \widetilde{K} d A_{g_{\alpha}}<0
$$

and

$$
\int_{M} h_{\alpha} d A_{0} \rightarrow \int_{M}|\widetilde{K}| d A_{g_{\bar{\alpha}}}>0 \quad \text { as } \alpha \rightarrow \bar{\alpha}
$$


Then

$$
\begin{aligned}
\Delta W^{+}+\widetilde{K}_{\alpha} e^{2 W^{+}}-c_{\alpha} & =\frac{\left[a_{\alpha} C_{\alpha}-2 \pi \chi(M, \alpha)\right] h_{\alpha}}{\int_{M} h_{\alpha} d A_{0}}+a_{\alpha} \widetilde{K}_{\alpha}\left(e^{2 a_{\alpha} W_{\alpha}}-1\right) \\
& \leq \frac{a_{\alpha} h_{\alpha}}{\int_{M} h_{\alpha} d A_{0}}\left[C_{\alpha}+2 \pi n a_{\alpha}+\left|e^{2 a_{\alpha} W_{\alpha}}-1\right| \int_{M} h_{\alpha} d A_{0}\right] \leq 0,
\end{aligned}
$$

as $\alpha \rightarrow \bar{\alpha}$, which proves that $W^{+}$is a bounded upper solution. From Lemma 1 , it follows that there exists a solution $W \in H_{2}^{p}(M) \cap C^{\infty}(\widehat{M})$ of equation (2).

Proof of Corollary. Note that under the stereographic projection $\mathbf{P}: \mathbf{R}^{2} \rightarrow$ $S^{2} \backslash\{p\}, 4|x|^{-1} \approx \operatorname{dist}(\mathbf{P} x, p)$ as $|x| \rightarrow \infty$ and the Euclidean metric on $\mathbf{R}^{2}$ can induce a flat conformal metric on $S^{2}$ representing a divisor $\bar{\alpha}:=-2 p$ with $\chi\left(S^{2}, \bar{\alpha}\right)=\chi\left(S^{2}\right)-2=0$. Thus, under this projection, by translating Theorems $\mathrm{A}$ and $\mathrm{B}$ on $S^{2}$ into $\mathbf{R}^{2}$, we have this corollary.

\section{ACKNOWLEDGMENTS}

The author wishes to thank R. McOwen for bringing this problem to his attention and giving very useful suggestions. Also, he thanks $M$. Troyanov for his comments.

\section{REFERENCES}

[A] P. Aviles, Conformal complete metrics with prescribed nonnegative Gaussian curvature in $\mathbf{R}^{2}$, Invent. Math. 83 (1986), 519-544.

[CN] K.-S. Cheng and W.-M. Ni, On the structure of the conformal Gaussian curvature equation on $\mathbf{R}^{2}$, Duke Math. J. 62 (1991), 721-737.

[HT] D. Hulin and M. Troyanov, Prescribing curvature on open surfaces, preprint.

[K] J. Kazdan, Prescribing the curvature of a Riemannian manifold, CBMS-NSF Regional Conf. Ser. in Math., vol. 57, 1985.

[KW] J. Kazdan and F. Warner, Curvature functions for compact 2-manifolds, Ann. of Math. (2) 99 (1974), 14-47.

[M1] R. McOwen, Prescribed curvature and singularities of conformal metrics on Riemann surfaces, J. Math. Anal. Appl. (to appear).

[M2] - Conformal metrics in $\mathbf{R}^{2}$ with prescribed Gaussian curvature and positive total curvature, Indiana Univ. Math. J. 34 (1985), 97-104.

[M3] - On the equation $\Delta u+K(x) e^{2 u}=f$ and prescribed negative curvature on $\mathbf{R}^{2}, \mathrm{~J}$. Math. Anal. Appl. 103 (1984), 365-370.

[M4] _ Point singularities and conformal metrics on Riemann surfaces, Proc. Amer. Math. Soc. 103 (1988), 222-224.

[N] W.-M. Ni, On the elliptic equation $\Delta u+K(x) e^{2 u}=0$ and conformal metrics with prescribed Gaussian curvature, Invent. Math. 66 (1982), 343-352.

[T] M. Troyanov, Prescribing curvature on compact surfaces with conical singularities, Trans. Amer. Math. Soc. 324 (1991), 793-821. 